\title{
EXPONENTIAL LAW FOR UNIFORMLY CONTINUOUS PROPER MAPS
}

\author{
R. Ayala, E. Dominguez, A. Quintero
}

\begin{abstract}
The purpose of this note is to prove the exponential law for uniformly continuous proper maps.
\end{abstract}

Let $X$ be a regular space and $Y$ a locally compact regular space. It is well known that the spaces of continuous maps $C(X \times Y, Z)$ and $C(X, C(Y, Z))$ are homeomorphic considering the compact-open topology. This property has important consequences in the study of the path-components of the function spaces and in Homotopy Theory. The exponential law for the uniformly continuous proper maps has similar consequences in some particular cases.

All the spaces we consider, unless otherwise mentioned, are metric spaces. A proper map will be a continuous map $f: X \longrightarrow Y$ such that for every compact subspace $K$ of $Y, \quad f^{-1}(K)$ is a compact in $X$. To abbreviate; we will say that $f$ is a $p$-map. A $u$-map is a uniformly continuous map, and a $u p$-map will be a uniformly continuous $p$-map. A up-isomorphism $f$ is a homeomorphism such that $f$ and $f^{-1}$ are $u$-maps. By $C(X, Y), \quad C_{p}(X, Y)$ and $C_{u p}(X, Y)$ we will denote the sets of continuous maps, $p$-maps and up-maps between $X$ and $Y$, respectively. With $C_{u p}^{u}(X, Y)$ we wilf represent the space of $u p$-maps with the topology of uniform convergence.

In this note we prove that the up-maps follow the exponential law if $X$ is compact; that is, the functors $X \times(-)$ and $C_{u p}^{u}(X,-)$ are adjoint. We also prove that if $X$ is not compact these functors are not generally adjoint.

A up-homotopy ( $p$-homotopy) between up-maps (p-maps) is a homotopy which is a up-map (p-map). With $[-,-],[-,-]_{p}$, and $[-,-]_{\text {up }}$ we will represent the sets of homotopy, $p$-homotopy and up-homatopy respectively. Also, the corresponding homotopy classes will be denoted by $[f],[f]_{p}$ and $[f]_{\mathrm{up}} . \mathrm{R}^{\mathrm{n}}$ will stand for the $n$-dimensional Euclidean space, and $I$ for the unit interval $\{0,1]$ with that distance. The Euclidean norm will be represented by $|-|$, and the distance of a metric space by $d(-,-)$. 
Theorem. Let $X, Y, Z$ metric spaces. We can define an injective map

$$
\Phi: C_{u p}(X \times Y, Z) \longrightarrow C_{u p}\left(X, C_{u p}^{u}\{Y, Z)\right)
$$

as $\Phi(f)(x)(y)=f(x, y)$. If $X$ is compact, then $\Phi$ is onto.

Proof: It is easy to see that:

(a) For each $x \in X$ the map $\Phi(f)\{x\}$ is a $u p$-map, because it is the composition of two up-maps.

(b) $\Phi(f)$ is a $u$-map, because $f$ is a $u$-map.

(c) Let us see that $\Phi(f)$ is a $p$-map. Given a compact subspace $K \subset$ $C_{\text {up }}^{u}(Y, Z)$, we only have to prove that any sequence $\left\{x_{n}\right\}$ in $\Phi(f)^{-1}(K)$ has a cluster point. Let $\left\{z_{n}\right\}$ be a subsequence of $\left\{x_{n}\right\}$ such that $\left\{\Phi(f)\left(z_{n}\right)\right\}$ is convergent; let $\theta \in K$ be the limit point. Then, for each $y_{0} \in Y$ the sequence $\left\{f\left(z_{n}, y_{0}\right)\right\}$ converges to $\theta\left(y_{0}\right)$. Consequently, $H=\left\{f\left(z_{n}, y_{0}\right) ; n \in \mathbf{N}\right\} \cup\left\{\theta\left(y_{0}\right)\right\}$ is a compact subspace of $\mathbf{Z}$. This implies that the sequence $\left\{x_{n}\right\}$ has a cluster point.

Hence $\Phi$ is well defined and it is an injective map. Since $X$ is compact, each continuous map defined on $X$ is also uniformly continuous. Then, given a continuous map

$$
g: X \longrightarrow C_{u p}^{U}(Y, Z)
$$

it is enough to show that $f: X \times Y \longrightarrow Z$ defined as $f(x, y)=g(x)(y)$ is a up-map.

To prove that $f$ is a $u$-map, let $f_{x}: Y \longrightarrow Z$ and $f_{v}: X \longrightarrow Z$ the maps defined by $f_{x}(y)=f(x, y)=f_{y}(x)$ for each couple $(x, y)$. According to [1, X.2.1.2] it suffices to show that the sets

$$
H=\left\{f_{x} ; x \in X\right\} \text { and } C=\left\{f_{y} ; y \in Y\right\}
$$

are uniformly equicontinuous. But $H=g(X)$ is a compact subset of $C_{u p}^{u}(Y, Z)$, hence it is uniformly equicontinuous by the theorem of Ascoli (see $[1, X .2 .5 .2]$ ). Since $g$ is a $u p$-map, it can be easily shown that $C$ is uniformly equicontinuous set.

It remains to show that $f$ is a $p$-map. Let $K$ be a compact subset of $\mathbf{Z}$. If $M=$ $\bigcup\left\{g(x)^{-1}(K) ; x \in X\right\}$, it is easy to check that $f^{-1}(K) \subset X \times M$. It suffices to prove that $M$ is a compact subset of $Y$. Given a sequence $\left\{y_{n}\right\}$ in $M$, there is a sequence $\left\{x_{n}\right\} \subset X$ such that $g\left(x_{n}\right)\left(y_{n}\right) \in K$, for each $n \in N$. Because $X$ and $K$ are compact, we can assume that $\left\{x_{n}\right\}$ and $\left\{g\left(x_{n}\right)\left(y_{n}\right)\right\}$ converge to $x_{0} \in X$ and $z_{0} \in K$ respectively. Then, $\left\{g\left(x_{n}\right)\right\}$ converges to $g\left(x_{0}\right)$, and it is obvious that for each $\epsilon>0$ there exists $n_{0}$ such that $d\left(z_{0}, g\left(x_{0}\right)\left(y_{n}\right)\right)<\epsilon$ if $n \geq n_{0}$. Therefore,

$$
\tilde{K}=\left\{g\left(x_{0}\right)\left(y_{n}\right) ; n \in \mathbf{N}\right\} \cup\left\{z_{0}\right\}
$$


is compact. Since $g\left(x_{0}\right)$ is proper, $g\left(x_{0}\right)^{-1}(\tilde{K})$ is also compact and $\left\{y_{n}\right\} \subset$ $g\left(x_{0}\right)^{-1}(\tilde{K})$ implies that $\left\{y_{n}\right\}$ has a cluster point. We conclude that $M$ is compact.

The following statement is easily proved:

Corollary. Let $X, Y, Z$ be metric spaces. Moreover, suppose that $X$ is compact. Then,

1) $\Phi: C_{u p}^{u}(X \times, Y, Z) \longrightarrow C_{u p}\left(X, C_{u p}^{u}(Y, Z)\right)$ is up-isomorphism.

2) The set of path-components of $C_{u p}^{u}(Y, Z)$ is in bijective correspondence with $[Y, Z]_{i p}$.

9) $\Phi$ induces a bijective correspondence

$$
\tilde{\Phi}:[X \times Y, Z]_{u p} \longrightarrow\left[X, C_{u p}^{u}(Y, Z)\right] .
$$

Remarks and Examples. If we consider only proper maps, the natural map $\Phi: C_{p}(X, Y, Z) \longrightarrow C_{p}\left(X, C_{p}(Y, Z)\right)$ where $C_{p}(Y, Z)$ is endowed with the compact-open topology, is easily checked to be well defined and injective. However, several troubles appear:

(1) The compactness of $X$ is necessary in order to prove that $\Phi$ is onto, even if $C_{p}(Y, Z)$ is endowed with the uniform convergence topology. The following example shows this fact:

Let $g: \mathbf{R} \longrightarrow C_{p}(I, \mathbf{R})=C(I, \mathbf{R})$ be the map given by $g(x)(t)=x t-(1-t) x^{3}$. It is easy to check the continuity of $g$. In order to prove that $g$ is proper, we take a compact $K \subset C(I, \mathbf{R})$ and a sequence $\left\{x_{n}\right\} \subset g^{-1}(K)$. Then there exists a subsequence $\left\{z_{n}\right\}$ of $\left\{x_{n}\right\}$, such that $\left\{g\left(z_{n}\right)\right\}$ converges to $\theta \in K$. In particular, $\lim g\left(z_{n}\right)(1)=\lim z_{n}=\theta(1)$ and we conclude that $g \in C_{p}(\mathbf{R}, C(I, \mathbf{R}))$. But the continuous map $f(x, t)=g(x)(t)$ is not proper because $\left(\left(\frac{t}{1-t}\right)^{\frac{1}{2}}, t\right) \in f^{-1}(0)$ for each $t \in[0,1)$.

(2) Although $X$ is compact, we cannot ensure that $\Phi$ is onto if we consider the compact-open topology on $C_{p}(Y, Z)$ :

Let $g: I \longrightarrow C_{p}(\mathbf{R}, \mathbf{R})$ given by $g(0)(x)=g_{0}(x)=x$ and

$$
g(t)(x)=g_{t}(x)=\left\{\begin{array}{ll}
x & \text { if } 0 \leq x \leq 1 / 2 t \\
-x+1 / t & \text { if } 1 / 2 t \leq x
\end{array} \quad(0<t \leq 1)\right.
$$

It is clear that $g_{t} \in C_{p}(\mathbf{R}, \mathbf{R})$ for each $t \in I$. The continuity of $t \longmapsto g_{t}$ follows from the fact that $\lim t_{n}=t_{0}$ in $I$ implies that $\left\{g_{t_{n}}\right\}$ converges uniformly on the compact subsets of $\mathbf{Z}$ to $g_{t_{0}}$. But $f(t, x)=g_{t}(x)$ is not a proper map because $(t, 1 / t) \in f^{-1}(0)$ for each $t \in(0,1]$.

(3) The proof of the Theorem assures that $\Phi$ is onto if we consider the uniform convergence topology, $u$, on $C_{\mathrm{p}}(Y, Z)$ and we assume the compactress of $X$. But in such situation,

$$
\Phi: C_{p}(X \times Y, Z) \rightarrow C\left(X, C_{p}^{u}(Y, Z)\right)
$$


is not well defined as shows the next example:

Let $f: I \times \mathbf{R} \longrightarrow \mathbf{R}^{3}$ be the map $f(t, x)=(t, x, t x)$. This map is a $p$-map, and if $\Phi(f)$ was continuous and $t_{n} \longrightarrow t_{0}$, given $\epsilon>0$ there would exist $n_{0} \in N$ such that if $n \geq n_{0}$

$$
|x|\left|t_{n}-t_{0}\right| \leq\left|\left(t_{n}, x, t_{n} x\right)-\left(t_{0}, x, t_{0} x\right)\right|<\epsilon
$$

for each $x \in \mathbf{R}$. Taking $|x|$ large enough would yield the contradiction $\epsilon<$ $|x|\left|t_{n}-t_{0}\right|<\epsilon$.

The next proposition shows that there is not any possible duality up-isomorphism when $X$ is not compact.

Proposition. Let $Z$ be the open interval $(-1,1)$. There exists no up-homotopy equivalence between $C_{u p}^{u}\left(\mathbf{R}^{2}, \mathbf{Z}\right)$ and $C_{u p}^{u}\left(\mathbf{R}, C_{u p}^{u}(\mathbf{R}, Z)\right)$.

We will need the following lemma:

Lemma. The map $\lambda:\left[\mathbf{R}^{n}, Z\right]_{u p} \longrightarrow\left[\mathbf{R}^{n}, Z\right]_{p}$ given by $\left.\lambda(\mid f]_{u p}\right)=[f]_{p}$ is bijective.

Proof: If $f, g: \mathbf{R}^{n} \longrightarrow Z$ are $p$-homotopic $u p$-maps, the homotopy $H: \mathbf{R}^{n} \times$ $I \longrightarrow Z$ given by $H(x, t)=t f(x)+(1-t) g(x)$ is a $u$-map. Now, we are going to show that $H$ is proper: Let $K \subset Z$ be a compact subset and $\left\{v_{n}=\left(x_{n}, t_{n}\right)\right\}$ a sequence in $H^{-1}(K)$. We may assume that $\left\{t_{n}\right\}$ converges to $t_{0}$. If we suppose that $\left\{v_{n}\right\}$ has no cluster points we have $\lim x_{n}=\infty$. Because $f$ and $g$ are $p$-homotopic we get $\lim f\left(x_{n}\right)=\lim g\left(x_{n}\right) \in\{-1,1\}$. If this common limits is 1 and $U$ is an euclidean neighbourhood of 1 missing $K$, there exists $n_{0}$ such that $f\left(x_{n}\right), g\left(x_{n}\right) \in U$ for each $n \geq n_{0}$. In particular, $H\left(x_{n}, t_{n}\right) \in U \quad\left(n \geq n_{0}\right)$ contradicts the assumption $\left\{\left(x_{n}, t_{n}\right)\right\} \subset H^{-1}(K)$. So, $\lambda$ is an injective map.

In order to prove that $\lambda$ is onto we recall that $\left[\mathbf{R}^{n}, Z\right]_{p}=\left[S^{n-1}, S^{0}\right]$, and its elements are the $p$-classes of the maps $g_{-1}, g_{1}: \mathbf{R}^{n} \longrightarrow Z$ given by $g_{j}(v)=$ $|v| j /(1+|v|) \quad(j=-1,1)$ if $n \geq 2$, or $h(v),|h(v)|,-h(v),-|h(v)|$ if $n=1$, where $h(v)=(2 / \pi) \arctan (v)$. This follows, for instance, from the embedding theorems of Edwards-Hastings, see $[2,6.2 .7]$. Now, $\lambda$ is onto because all the representatives are up-maps.

Proof of Proposition 4: It suffices to prove that those spaces have not the same number of path-components. As a consequence of Corollary 2.2 ) the pathcomponents of $C_{u p}^{U}\left(\mathbf{R}^{2}, Z\right)$ are in bijective correspondence with $\left[\mathbf{R}^{2}, Z\right]_{s p}$. But $\left[\mathbf{R}^{2}, Z\right]_{u p} \simeq\left[\mathbf{R}^{2}, Z\right]_{p}$, by lemma 5 , and the latter set has two elements.

Now, we are going to show that $C_{u p}^{u}\left(\mathbf{R}, C_{u p}^{u}(\mathbf{R}, Z)\right)$ has at least four pathcomponents. As above, $C_{u p}^{u}(\mathbf{R}, Z)$ has four path-components, and they are the components of $g_{0}(x)=(2 / \pi) \arctan (x), \quad g_{1}=-g_{0}, g_{2}=\left|g_{0}\right|$ and $g_{3}=$ $=-\left|g_{0}\right|$. Since $C_{u p}^{u}(\mathbf{R}, Z)$ is metrizable and $g_{0}$ is a $u$-map, $f_{0}: \mathbf{R} \longrightarrow$ $C_{u p}^{u}(\mathbf{R}, Z)$ defined by $f_{0}(t)(x)=g_{0}(t+x)$ is a $u$-map. Also, by using the 
theorem of Ascoli it is easy to check that $f_{0}$ is a $p$-map. So, we have got a $u p$-map $f_{0}$ such that $f_{0}(\mathbf{R})$ lies in the path-component of $g_{0}$. In a similar way, we get $u p$-maps $f_{i}$ with $f_{i}(\mathbf{R})$ lying in the path-component of $g_{i} \quad(i=1,2,3)$. In particular, $f_{i}$ and $f_{j}$ are not up-homotopic $(0 \leq i \neq j \leq 3)$. We conclude, applying corollary 2.2) again, that the $u p$-maps $\left\{f_{i}\right\}_{0 \leq i \leq 3}$ define four distinct path-components.

Remark. If the metric on $Z$ is not bounded, lemma 5 is false. Indeed, for each pair of real numbers $a_{1}, a_{2}>0$, the up-maps $f_{1}, f_{2}: \mathbf{R} \longrightarrow \mathbf{R}$ given by $f_{i}(t)=a_{i} t \quad(i=1,2)$, are $p$-homotopic, but not $u p$-homotopic: If $H: \mathbf{R} \times I \longrightarrow$ $\mathbf{R}$ is a $u p$-homotopy between $f_{1}$ and $f_{2}$, by $[3$, III.10] there would exist $\epsilon>0$ such that

$$
\left|H(x, t)-H\left(y, t^{\prime}\right)\right| \leq \max \left\{\epsilon\left|(x, t)-\left(y, t^{\prime}\right)\right|, \epsilon\right\}
$$

for each couple $(x, t),\left(y, t^{t}\right) \in \mathbf{R} \times I$. Therefore,

$$
\left|a_{1}-a_{2}\right| x=\left|f_{1}(x)-f_{2}(x)\right|=|H(x, 0)-H(x, 1)| \leq \epsilon
$$

and taking $x$ large enough the above inequality would yield the contradiction $\epsilon<x\left|a_{1}-a_{2}\right|<\epsilon$.

In fact, we have proved that $\operatorname{card}[\mathbf{R}, \mathbf{R}]_{u_{p}} \geq \operatorname{card} \mathbf{R}$.

\section{References}

1. N. BoURBaKI, in "General Topology," Hermann, 1966.

2. D.A. EDWARDS, H.M. HASTINGS, Cech and Steenrod Homotopy Theory with applications to Geometric Topology, Lect. Notes 542 Springer (1976).

3. J.R. ISBELL, Uniform spaces, Math. Surveys, 12 AMS (1964).

R. Ayala: Dpto. de Geometría y Topología

Facultad de Matemáticas

41012-Sevilla, SPAIN.

E. Dominguez: Dpto. de Matemáticas

Facultad de Ciencias

Ciudad Universitaria

50009-Zaragoza, SPAIN.

A. Quintero: Dpto. de Geometría y Topologf́a

Facultad de Matemáticas

41012-Sevilla, SPAIN.

Rebut el 30 de Jury de 1987 